\title{
AVALIAÇÃO DA INCORPORAÇÃO DE RESÍDUO DE CORTE DE MÁRMORE E GRANITO EM CONCRETO PARA PRODUÇÃO DE PISOS INTERTRAVADOS PARA PAVIMENTAÇÃO
}

\author{
EVALUATION OF THE INCORPORATION OF MARBLE AND GRANITE \\ CUTTING RESIDUES IN CONCRETE FOR THE PRODUCTION OF \\ INTERLOCKING PAVING FLOORS
}

\author{
${ }^{1}$ Wandercleiton Cardoso* \\ ${ }^{2}$ Raphael Colombo Baptista \\ ${ }^{3}$ Thiago Augusto Pires Machado \\ ${ }^{4}$ André Gustavo de Sousa Galdino \\ ${ }^{5}$ Renzo di Felice
}

\footnotetext{
${ }^{1}$ Università degli Studi di Genova. E-mail: wandercleiton.cardoso@dicca.unige.it ${ }^{2}$ Instituto Federal do Espírito Santo. E-mail: colombo.raphael@hotmail.com ${ }^{3}$ Instituto Federal do Espírito Santo. E-mail: thiagoaugustopn@yahoo.com.br

${ }^{4}$ Instituto Federal do Espírito Santo. E-mail: andregsg@ifes.edu.br

${ }^{5}$ Università degli Studi di Genova. E-mail: renzo.difelice@unige.it

*Autor de correspondência
}

Artigo submetido em 08/05/2021, aceito em 16/07/2021 e publicado em 31/08/2021.

Resumo: A indústria da construção civil é uma atividade que consome elevado volume de recursos naturais e no cenário atual é imprescindível se preocupar com o desenvolvimento sustentável e encontrar alternativas de reaproveitamento de resíduos sólidos. Nesse contexto, a reinserção do resíduo de corte de mármore e granito (RCMG) na cadeia produtiva é uma alternativa para amenizar um sério problema ambiental, pois o consumo de rochas ornamentais vem crescendo e elevando a quantidade de resíduo produzido. Devido ao grande volume de resíduos de corte de granito produzido e não reutilizado, este artigo técnico avalia a viabilidade técnica da sua utilização como adição em concretos e produção de blocos intertravados com resistência à compressão mínima de $35 \mathrm{MPa}$ e a absorção d'água de $6 \%$ no máximo. As matérias primas foram caracterizadas quanto à massa específica, massa unitária, distribuição do tamanho de partículas, materiais pulverulentos e composição química (FRX). Em seguida, os corpos de prova foram moldados de acordo com as prescrições da norma técnica NBR 9781:2013 e foram realizadas análises de desempenho mecânico (resistência à compressão) e absorção d'água. Em suma, a partir dos resultados obtidos no programa experimental, o uso do resíduo de corte de mármore e granito como adição em concretos para produção de pisos de pavimentação é viável tecnicamente para tráfego de pedestres, veículos leves e veículos comerciais de linha

Palavras-chave: granito; mármore; concreto; propriedades químicas; propriedades mecânicas. 


\begin{abstract}
The construction industry is an activity that consumes a high volume of natural resources and in the current scenario it's essential to be concerned with sustainable development and find alternatives for reusing solid waste. In this context, the reinsertion of marble and granite cutting waste in the production chain is an alternative to alleviate a serious environmental problem, as the consumption of ornamental stones has been growing and increasing the amount of waste produced. Due to the large volume of granite cutting waste produced and not reused, this technical article assesses the technical feasibility of its use as an addition to concrete and production of interlocking blocks with a minimum compressive strength of $35 \mathrm{MPa}$ and water absorption of no maximum $6 \%$ as provided for regulatory requirements. The raw materials were characterized in terms of specific mass, unit mass, particle size distribution, powdery materials and chemical composition. Then, the specimens were molded in accordance with the requirements of technical standard NBR 9781:2013 and analysis of mechanical performance (compression resistance) and water absorption were performed. It is concluded that from the results obtained in the experimental program, the use of marble and granite cutting waste, as an addition in concrete for the production of paving floors, is technically feasible for pedestrian traffic, light vehicles and commercial vehicles.
\end{abstract}

Keywords: granite; marble; concrete; chemical properties; mechanical properties.

\section{INTRODUÇÃO}

A indústria da construção civil é uma atividade que consome elevado volume de recursos naturais e no cenário atual é imprescindível se preocupar com o desenvolvimento sustentável e encontrar alternativas de reaproveitamento de resíduos sólidos, visto que atualmente existe um apreciável volume de resíduos com dificuldades relacionadas ao seu armazenamento, manejo, transporte $\mathrm{e}$ destinação final, além de ônus para o produtor e problemas ambientais.

Nesse contexto, a reinserção do resíduo de corte de mármore e granito (RCMG) na cadeia produtiva é uma alternativa para amenizar um sério problema ambiental (ARUNTAS et al., 2010; THAKUR et al., 2018; LI et al., 2018a; AWAD et al., 2020).

$\mathrm{Na}$ zona urbana se concentram os maiores aglomerados populacionais e as maiores extrações de recursos naturais, sendo a indústria de construção civil responsável por consumir $75 \%$ desses recursos no planeta (MEDINA et al., 2018).

No Brasil aproximadamente 210 milhões de toneladas de agregados naturais são utilizados anualmente na produção de argamassas e concretos, sendo que, essa gigantesca demanda esgota as reservas de matérias primas nas proximidades destes centros urbanos, aumentando o consumo de energia, a poluição, o custo produtivo e custos de transporte desses materiais a longas distâncias (MONTANI, 2017; CHIODI, 2018).

A indústria de rochas ornamentais é responsável por movimentar expressivos volumes no mercado nacional e internacional. A produção mundial noticiada de rochas ornamentais e de revestimento evoluiu de 1,8 milhões de toneladas ao ano na década de 1920 para 145 milhões de toneladas no ano de 2016, sendo que cerca de 53,5 milhões de toneladas de rochas brutas e beneficiadas foram comercializadas no mercado internacional em 2016, representando 790 milhões $\mathrm{m}^{2}$ equivalentes de chapas com 2 $\mathrm{cm}$ de espessura. (MONTANI, 2017; CHIODI, 2018).

Em 2016, no Brasil, foram produzidas 8,5 milhões de toneladas distribuídas entre mármores, travertinos, granitos e similares, gerando aproximadamente 2,1 milhões de toneladas de rejeitos oriundos desse processamento, ou seja, $25 \%$ de resíduo de corte de mármore e granito (CHIODI, 2018).

Durante o processo de beneficiamento das rochas, quando essas 
são cortadas em chapas de $20 \mathrm{~mm}$ de espessura, são gerados aproximadamente $20 \%$ a $30 \%$ de resíduos em forma de lama, ficando muitas vezes sem tratamento adequado, disponível a céu aberto, ou lançado em rios e córregos (CHIODI, 2018).

Os estados do Espírito Santo e Minas Gerais são os maiores produtores e exportadores brasileiros, seguidos pela Bahia e Ceará. Em 2016, o Brasil foi o $4^{\circ}$ maior produtor mundial, enquanto a Itália ocupou a $6^{\circ}$ posição com uma produção com uma produção $26 \%$ menor quando comparado com a produção do Brasil, totalizando 6,52 milhões de toneladas (CHIODI, 2018).

As empresas italianas do setor de rochas ornamentais (mármore) concentram-se principalmente nas regiões tradicionais de Lucca, Massa, Carrara e Verona. O porto de Carrara, equipado com instalações especiais para carga e descarga e depósito de pedra, constitui-se em elemento importante da infraestrutura de produção e comercialização desse setor, uma vez que por ele circula quase metade do comercio comércio exterior referente a rochas ornamentais (MONTANI, 2017; CHIODI, 2018).

O consumo de rochas ornamentais vem crescendo e, consequentemente, elevando-se a quantidade de resíduo gerada. Este volume expressivo de resíduos demonstra a importância de aproveitá-lo, na construção civil, pois apresenta grande abrangência, consome grandes quantidades de insumos naturais e, além disso, o RCMG possui elevada finura, sendo bastante compatível com as matrizes cimentícias, podendo ser utilizado como filler em argamassas e concretos (AYDIN et al., 2019; BAGHEL et al., 2020; JAIN et al., 2020).

Neste contexto, devido ao grande volume de resíduos de corte de granito produzido e não reutilizado, este artigo técnico avalia a viabilidade técnica da sua utilização como adição em concretos e produção de blocos intertravados com resistência à compressão mínima de 35 $\mathrm{MPa}$ e a absorção d'água de no máximo a $6 \%$, conforme previsto na norma técnica NBR 9781 (ABNT, 2013).

\section{REFERENCIAL TEÓRICO}

$\mathrm{O}$ beneficiamento de mármores e granitos tem como objetivo a transformação dos blocos, extraídos na fase de lavra, em produtos finais ou semiacabados.

$\mathrm{Na}$ fase de lavra os blocos são extraídos em grandes dimensões, enquanto no beneficiamento primário os blocos são preparados e serrados em chapas de espessura variável, usualmente dois ou três centímetros e são submetidas a acabamento superficial, com ou sem resinagem, que pode ser um simples desbaste, polimento, escovado e flameado e na etapa de beneficiamento final tem-se a produção de ladrilhos (AWAD et al., 2019a; AZEVEDO et al., 2019).

Os mármores e granitos são largamente utilizados na arquitetura e na indústria de construção civil, entretanto, mármores e granitos são apenas alguns dos tipos de rochas utilizadas na construção civil. As rochas são agregados naturais sólidos, compostos de um ou mais minerais, classificadas em três grupos: magmáticas, metamórficas e sedimentares (LI et al., 2019; MATOS et al., 2020; NASCIMENTO et al., 2020).

As rochas magmáticas são resultantes da consolidação do magma na crosta terrestre, podendo ser intrusiva ou extrusiva. As rochas magmáticas intrusivas ocorrem quando a consolidação se faz lentamente na subsuperfície terrestre, tendo tempo suficiente para os minerais crescerem e serem facilmente visíveis, como é o caso do granito, composto por quartzo, feldspatos, micas e anfibólios (LI et al., 2018b; ZANELATO et al., 2020; SALMAN et al., 2021). 
Entretanto, quando a lava vulcânica se consolida na superfície terrestre se resfria rapidamente não permitindo que os cristais se desenvolvam, originando as rochas magmáticas extrusivas, como $\mathrm{o}$ caso do basalto. As rochas magmáticas escuras são ricas em minerais como magnésio e ferro, enquanto que as rochas claras são ricas em silício e alumínio.

Para a produção de concreto e argamassas é necessário conhecer a composição química do granito, pois, elementos como por exemplo, o magnésio, pode gerar expansibilidade no estado endurecido, refletindo em fissuras quando utilizadas em revestimentos e pavimentação. Além disso, a finura do resíduo e o teor de $\mathrm{SiO}_{2}$ associado ao processo produtivo pode apresentar elevada reatividade, tendo como consequência a ocorrência de reações álcali-agregado (LI et al., 2019; MESSAOUDA et al., 2019).

As rochas metamórficas são provenientes da transformação de rochas preexistentes, mediante condições de temperatura e pressão e dependendo dos eventos geológicos de cada região, como cadeias montanhosas, sua estrutura é diferenciada. O mármore, exemplo de rocha metamórfica é composto principalmente por calcita (BINCI et al., 2007; TORRES et al., 2009).

Referente às rochas sedimentares, resultantes da deposição de detritos de outras rochas, magmáticas ou metamórficas, ou do acúmulo de detritos orgânicos ou, ainda, da precipitação química, tem-se como exemplo o arenito e o calcário (BILGIN et al., 2012).

Durante a extração as rochas são cortadas em chapas para o uso em vários ramos da construção civil, gerando resíduos que se caracterizam pelo tipo da rocha extraída, como mármores, granitos, arenitos e outras rochas. (INCE et al., 2020)
O ciclo produtivo de rochas ornamentais compreende três etapas básicas: a extração ou mineração das rochas com dimensões aproximadas de 3,0 x 2,0 x 1,5 m, seguido pelo beneficiamento primário, que consiste na serragem dos blocos utilizando teares para produzir chapas com medidas aproximadas de 2,80 $\mathrm{x} 1,90 \mathrm{x} 0,02 \mathrm{~m}$ e finalizando o ciclo produtivo tem-se $\mathrm{o}$ beneficiamento secundário, que ocorre nas indústrias de transformação final, onde são realizados os acabamentos conforme solicitação dos clientes (MONTANI, 2017).

$\mathrm{Na}$ indústria de beneficiamento primário para cada metro cúbico de rocha serrado nos teares são produzidos em média, $32 \mathrm{~m}^{2}$ de chapas com $2 \mathrm{~cm}$ de espessura ou $49 \mathrm{~m}^{2} \mathrm{com} 1 \mathrm{~cm}$ de espessura.

Os teares utilizados são de fios diamantados ou de lâminas metálicas, sendo que no primeiro caso o resíduo é constituído por pó de rocha e água, mas quando utilizado o tear de lâminas metálicas, o tipo mais difundido, a lama gerada é formada por água, cal, granalha e fragmentos metálicos das próprias lâminas.

Essa lama escoa até o poço de recolhimento, é recirculada pelos teares através de bombas centrifugas e é continuamente aspergida na superfície de corte, para desobstruir os canais entre as chapas e facilitar a serragem. Além de lubrificar e resfriar as lâminas metálicas, evitando a sua oxidação (MAHESH et al., 2021).

Os resíduos gerados pelo corte das rochas também são gerados na indústria extrativa (pedreiras), e depois, após a extração dos blocos, esses são novamente serrados nos teares na etapa de beneficiamento primário e, posteriormente, quando as chapas são trabalhadas na etapa de beneficiamento secundário (marmorarias), onde chapas brutas são divididas em partes menores, ou seja, resíduos de mármore e granito são produzidos em toda a cadeia produtiva. 
Durante o beneficiamento secundário (marmorarias) as serras utilizadas são de lâminas de disco metálico diamantado e o resíduo gerado é diversificado, portanto, sua caracterização física, química e mineralógica é imprescindível para uso em novos produtos (MARTINI et al., 2018).

Referente ao tratamento do resíduo gerado na cadeia produtiva oriundo da fração mais viscosa da lama, normalmente é encaminhado para lagoas de decantação, ou a filtros prensa, com o objetivo de reduzir sua umidade.

Em seguida, este resíduo é transportado e descartado em aterros sem nenhum tratamento prévio e, em inúmeros casos, estes rejeitos também são depositados em rios, córregos ou esgotos provocando assoreamento de rios, contaminação de lençóis freáticos e gerando conflitos entre os órgãos ambientais e as populações vizinhas (MASHALY et al., 2018).

Para minimizar os impactos ambientais, as empresas constroem unidades de tratamento de efluentes, como caixas de decantação, e, assim, o resíduo pode ser utilizado para diversos fins, como, por exemplo, matéria prima de concretos e argamassas.

A composição química do resíduo de corte de mármore e granito (RCMG) é diversificada, e no geral, o resíduo é formado, por sílica $\left(\mathrm{SiO}_{2}\right)$, alumina $\left(\mathrm{Al}_{2} \mathrm{O}_{3}\right)$, cal $(\mathrm{CaO})$ e óxidos alcalinos $\left(\mathrm{Na}_{2} \mathrm{O}, \quad \mathrm{K}_{2} \mathrm{O}\right)$, contudo, encontra-se também o óxido de ferro $\left(\mathrm{Fe}_{2} \mathrm{O}_{3}\right)$, que pode aparecer em maior ou menor quantidade devido ao desgaste das lâminas de corte (GHALEHNOVI et al., 2019).

A Tabela 1 ilustra os compostos químicos, oriundos do corte de mármores e granitos. As siglas significam, respectivamente: Resíduo de Mármore (RM), Resíduo de Granito (RG), Resíduo de Mármores e Granitos (RMG), Resíduo de Rochas Ornamentais (RRO), Resíduo de Corte de Mármore (RCM), Resíduo do Corte de Granito (RCG), Resíduo de Corte de Mármore e Granito (RCMG), Resíduo de Beneficiamento de Mármore e Granito (RBMG) e Resíduo Beneficiamento de Rochas Ornamentais (RBRO).

A produção mundial noticiada de rochas ornamentais, incluindo os mármores e os granitos evoluiu de 1,8 Mt/ano, na década de 1920, para o patamar de 145 Mt/ano no ano de 2016 com cerca de 53,5 milhões de toneladas de rochas brutas e beneficiadas comercializadas no mercado internacional, representando 790 milhões $\mathrm{m}^{2}$ equivalentes de chapas com $2 \mathrm{~cm}$ de espessura.

A Tabela 2 apresenta os principais países produtores, exportadores e importadores mundiais. Analisando a Tabela 2 observa-se que a China foi o principal produtor mundial de rochas em 2016 e países como o Brasil, $4^{\circ}$ maior produtor e a Itália, $6^{\circ}$ maior produtor, sendo responsáveis por mais de $10 \%$ da produção mundial de rochas ornamentais.

Observa-se também um aumento significativo na produção mundial com exponencial justificada pela grande variedade de rochas ornamentais presentes principalmente em países como China, Índia, Brasil e Itália, como mármore, granito, ardósia, quartzito e travertinos (MONTANI, 2017; CHIODI, 2018).

Diante deste cenário, as indústrias de rochas ornamentais durante seu processo de extração e beneficiamento produzem um grande volume de resíduos, sendo que, durante a etapa de beneficiamento dos blocos, cerca de 25 a $30 \%$ do seu volume é transformado em resíduo. 
Tabela 1: Composição química dos resíduos de mármore e granito (\%)

\begin{tabular}{|c|c|c|c|c|c|c|c|c|c|}
\hline Compostos & $\begin{array}{c}\text { RM } \\
\text { (1) }\end{array}$ & $\begin{array}{l}\text { RG } \\
(2)\end{array}$ & $\begin{array}{c}\text { RMG } \\
\text { (3) }\end{array}$ & $\begin{array}{c}\text { RRO } \\
\text { (4) }\end{array}$ & $\begin{array}{c}\text { RCM } \\
(5)\end{array}$ & $\begin{array}{c}\text { RCG } \\
(6)\end{array}$ & $\begin{array}{c}\text { RCMG } \\
\text { (7) }\end{array}$ & $\begin{array}{c}\text { RBMG } \\
(8)\end{array}$ & $\begin{array}{c}\text { RBRO } \\
(9)\end{array}$ \\
\hline $\mathrm{SiO}_{2}$ & 0.67 & 67.91 & 48.3 & 63.23 & 8.1 & 59.62 & 70.4 & 54.62 & 23.4 \\
\hline $\mathrm{Al}_{2} \mathrm{O}_{3}$ & 0.12 & 13.13 & 10.76 & 15.34 & 0.7 & 12.77 & 15.2 & 9.71 & 4.32 \\
\hline $\mathrm{Fe}_{2} \mathrm{O}_{3}$ & 0.08 & 1.97 & - & 3.53 & 0.7 & 9.49 & 2.0 & 5.86 & 2.83 \\
\hline $\mathrm{CaO}$ & 54.43 & 2.98 & 16.53 & 3.34 & 44.3 & 4.83 & 1.0 & 20.12 & 64.5 \\
\hline MgO & 0.59 & 0.51 & 0.78 & 0.34 & 5.6 & 1.96 & 0.35 & 3.22 & 1.73 \\
\hline $\mathrm{Na}_{2} \mathrm{O}$ & 0.14 & 4.69 & 3.73 & 3.04 & 0.01 & 2.72 & 3.7 & 2.15 & 0.79 \\
\hline $\mathbf{K}_{2} \mathbf{O}$ & 0.02 & 3.91 & 4.43 & 5.34 & 0.03 & 5.3 & 5.5 & 3.74 & 1.04 \\
\hline $\mathrm{SO}_{3}$ & 0.01 & 0.01 & 0.25 & 1.52 & 0.04 & 0.03 & 0.01 & 0.12 & 0.47 \\
\hline Outros & 0.54 & 1.98 & 3.49 & 2.98 & 0.02 & 1.36 & 084 & 0.06 & 0.62 \\
\hline \% Perda ao fogo & 43.4 & 2.91 & 11.73 & 1.34 & 40.5 & 1.92 & 1.0 & 1.0 & 0.30 \\
\hline
\end{tabular}

Fonte: (1) Aruntas et al. (2010); (2) Chen et al. (2020); (3) Awad et al. (2019b); (4) Azevedo et al. (2020);

(5) Aydin et al. (2019); (6) Gonçalves (2000); (7) Lopez et al. (2020); (8) Zanelato et al. (2020); (9) Ince et al. (2020).

Tabela 2: Principais produtores mundiais de rochas ornamentais (2013-2016)

\begin{tabular}{|c|c|c|c|c|c|c|c|c|}
\hline \multirow{2}{*}{$\begin{array}{l}\text { Países x Ano } \\
\text { (Peso) }\end{array}$} & \multicolumn{2}{|c|}{2013} & \multicolumn{2}{|c|}{2014} & \multicolumn{2}{|c|}{2015} & \multicolumn{2}{|c|}{2016} \\
\hline & Mt & $\%$ & Mt & $\%$ & Mt & $\%$ & Mt & $\%$ \\
\hline China & 39,5 & 30,4 & 42,5 & 31,1 & 45,0 & 32,1 & 46,0 & 31,7 \\
\hline Índia & 19,5 & 15,0 & 20,0 & 14,7 & 21,0 & 15,0 & 23,5 & 16,2 \\
\hline Turquia & 12,0 & 9,2 & 11,5 & 8,4 & 10,5 & 7,5 & 10,75 & 7,4 \\
\hline Brasil & 9,0 & 6,9 & 8,75 & 6,4 & 8,2 & 5,9 & 8,5 & 5,9 \\
\hline Irã & 6,5 & 5,0 & 7,0 & 5,1 & 7,5 & 5,4 & 8,0 & 5,5 \\
\hline Itália & 7,0 & 5,4 & 6,75 & 4,9 & 6,5 & 4,6 & 6,25 & 4,3 \\
\hline Egito & 3,0 & 2,3 & 4,2 & 3,1 & 5,0 & 3,5 & 5,25 & 3,6 \\
\hline Espanha & 5,0 & 3,8 & 4,85 & 3,6 & 4,75 & 3,4 & 5,0 & 3,4 \\
\hline EUA & 2,75 & 2,1 & 2,65 & 1,9 & 2,7 & 1,9 & 2,8 & 1,9 \\
\hline Portugal & 2,65 & 2,0 & 2,75 & 2,0 & 2,7 & 1,9 & 2,6 & 1,8 \\
\hline França & 1,05 & 0,8 & 1,2 & 0,9 & 1,25 & 0,9 & 1,3 & 0,9 \\
\hline Arábia Saudita & 1,2 & 0,9 & 1,3 & 1,0 & 1,2 & 0,9 & 1,25 & 0,9 \\
\hline Grécia & 1,25 & 1,0 & 1,3 & 1,0 & 1,25 & 0,9 & 1,2 & 0,8 \\
\hline Paquistão & 1,0 & 0,8 & 1,0 & 0,7 & 1,05 & 0,7 & 1,1 & 0,7 \\
\hline Subtotal & 111,4 & 85,6 & 115,75 & 84,8 & 118,6 & 84,6 & 123,5 & 85,0 \\
\hline Outros & 18,6 & 14,3 & 20,75 & 15,2 & 21,4 & 15,7 & 21,5 & 15,0 \\
\hline Total & 130,0 & 99,0 & 136,5 & 100 & 140,0 & 100,3 & 145,0 & 100 \\
\hline
\end{tabular}

Fonte: Montani (2017); Chiodi, (2018).

Somente no Brasil são gerados cerca de 800.000 t/ano de resíduo descartados inadequadamente no meio ambiente, sendo que somente algumas poucas empresas se preocupam em reduzir o impacto ambiental ao descartar estes resíduos no meio ambiente, ou seja, ainda não existe uma destinação sustentável para os resíduos de beneficiamento de rochas (MONTANI, 2017; CHIODI, 2018).

Conforme a literatura, diversos autores estudam metodologias de reutilizar o resíduo de beneficiamento de rochas ornamentais inserindo-os novamente na cadeia produtiva, como exemplo, produção de argamassas, produção de concretos, produção de blocos cerâmicos e produção de blocos estruturais (ERGÜN, 2011; KRSTULOVIĆ et al., 1994; LI et al., 2019).

Entre as diversas aplicações de reutilização do Resíduo de Corte de Mármore e Granito (RCMG) pode-se citar a produção de concreto para a produção de 
peças segmentadas para construção e pavimentação, como exemplos, blocos cerâmicos e pisos intertravados.

Países desenvolvidos como a Itália, por exemplo, não sofrem com problemas relacionados a pavimentação, entretanto, ainda são dependentes de produtos não renováveis, como o betume para pavimentação de vias públicas, entretanto, quando o assunto é o Brasil mais de $80 \%$ das vias nacionais não são pavimentadas (MONTANI, 2017; CHIODI, 2018).

A utilização de blocos para pavimentação se apresenta como uma solução para o problema, pois é possível utilizar RCMG para produção destes materiais, resolvendo assim este grave problema rural e urbano, além do fato que este tipo de pavimentação favorece a redução de inundações em cidades, pois o índice de infiltração das peças prémoldadas de concreto é maior e o índice de escoamento é menor quando comparados ao do asfalto, além do baixo custo de manutenção.

No Brasil a utilização de peças de pavimentação de concreto deve seguir o requisito normativo da NBR 9781 (ABNT, 2013). A Tabela 3 ilustra os valores de resistência mínima de acordo com o tipo de tráfego desejado.

Tabela 3: resistência característica à compressão

\begin{tabular}{|c|c|}
\hline Solicitação & $\begin{array}{l}\text { Resistência característica à } \\
\text { compressão aos } 28 \text { dias }\end{array}$ \\
\hline $\begin{array}{l}\text { Tráfego de pedestres, veículos leves e veículos comerciais } \\
\text { de linha }\end{array}$ & $\geq 35 \mathrm{MPa}$ \\
\hline $\begin{array}{l}\text { Tráfego de veículos especiais e solicitações capazes de } \\
\text { produzir efeitos de abrasão acentuados }\end{array}$ & $\geq 50 \mathrm{MPa}$ \\
\hline
\end{tabular}

Fonte: ABNT NBR 9781 (2013, p.6).

\section{MATERIAIS E MÉTODOS}

As matérias primas foram caracterizadas para se obter informações necessárias quanto às possibilidades de uso, principalmente o resíduo de corte de mármore e granito (RCMG) devido a sua composição ser desconhecida para este estudo.

As matérias primas foram caracterizadas quanto à massa específica, massa unitária, distribuição do tamanho de partículas, materiais pulverulentos e composição química (FRX).

Em seguida, os corpos de prova foram moldados de acordo com as prescrições das respectivas normas de ensaio e foram realizadas análises de desempenho mecânico e durabilidade em seus diferentes traços.

\subsection{Cimento}

O aglomerante cimentício utilizado foi o Cimento Portland de alta resistência inicial resistente a sulfatos (CPV ARI-RS). A finura foi determinada conforme a norma NBR 11579 "Cimento Portland Determinação do índice de finura por meio da peneira $75 \mu \mathrm{m} "(\mathrm{ABNT}, 2012)$.

A permeabilidade foi determinada conforme a norma NBR 16372 "Cimento Portland e outros materiais em pó Determinação da finura pelo método de permeabilidade ao ar método de Blaine" (ABNT, 2015). O início de fim de pega foi determinado conforme a norma NBR 16607 "Cimento Portland - Determinação dos tempos de pega" (ABNT, 2018).

A densidade real do cimento foi determinada pelo método do frasco de Le Chatelier conforme a norma NBR 16605 "Cimento Portland e outros materiais em pó - Determinação da massa específica" (ABNT, 2017).

O resíduo insolúvel foi determinado utilizando a NBR NM 15, o anidrido sulfúrico foi determinado utilizando a NBR 
NM 16 (ABNT, 2012), a perda ao fogo foi determinada utilizando a NBR NM 18 (ABNT, 2012), determinação de enxofre na forma de sulfeto utilizando a NBR NM 19 (ABNT, 2012), determinação de dióxido de carbono por gasometria utilizando a NBR NM 20 (ABNT, 2012), enquanto a composição química foi determinada através da técnica de espectroscopia por fluorescência de raios $\mathrm{X}$ (FRX) e com base na norma regulamentadora NBR NM 14 (ABNT, 2012).

\section{2 agregados}

O agregado miúdo proveniente de origem quartzosa foi previamente lavado e seco em estufa a uma temperatura aproximada de $105 \pm 1{ }^{\circ} \mathrm{C}$, peneirado na peneira \#6,3 $\mathrm{mm}$ e estocado em cubas cerâmicas antes dos ensaios e moldagens. A massa específica da areia foi determinada pelo método do frasco de Chapman conforme norma NBR 16916 "Agregado miúdo - Determinação da massa específica e massa específica aparente" (ABNT, 2021).

$\mathrm{O}$ agregado utilizado (gnaisse) de origem diabásica foi lavado, seco e peneirado, utilizando-se a fração passante na peneira $\# 19 \mathrm{~mm}$ e retida na peneira $\# 6,3 \mathrm{~mm}$. A massa específica da brita foi determinada pelo método da balança hidrostática conforme NBR 16917 "Agregado graúdo - Determinação da massa específica, massa específica aparente e absorção de água" (ABNT, 2021). Os agregados também foram ensaiados conforme norma NBR 7211 "Agregados para concreto - Especificação" (ABNT, 2019).

\subsection{Resíduo de corte de mármore e granito (RCMG)}

O resíduo utilizado proveniente da etapa de beneficiamento foi coletado em empresas de comercialização de rochas ornamentais e posteriormente foi exposto a radiação solar, seco em estufa a uma temperatura aproximada de $105 \pm 1^{\circ} \mathrm{C}$ e na sequência peneirado (finura de $75 \mu \mathrm{m}$ ), homogeneizado, quarteado e armazenado em local seco.

$$
\text { A composição química foi }
$$
determinada através da técnica de espectroscopia por fluorescência de raios $\mathrm{X}$ (FRX).

A fluorescência de raios $X(X R F)$ é uma técnica analítica que pode ser utilizada para determinar a composição química de uma ampla variedade de tipos de amostras, incluindo sólidos, líquidos, pastas e pós soltos. A fluorescência de raios $\mathrm{X}$ também é usada para determinar a espessura e a composição das camadas e revestimentos.

\subsection{Dosagem do concreto (corpos de prova)}

O concreto foi dosado com traço em massa de 1:0.8:1.2 (aglomerante, areia, brita 0 ) e relação água/aglomerante de 0,3 , sendo o aglomerante $=$ cimento $+\mathrm{RCMG}$, com abatimento do tronco de cone no estado fresco de $12 \pm 2 \mathrm{~cm}$.

Para melhorar a trabalhabilidade do concreto produzido foi adicionado 2,2 $\mathrm{kg} / \mathrm{m}^{3}$ do aditivo plastificante multifuncional de pega normal Muraplast FK 830 que possui a função de reduzir a tensão superficial da água de mistura e com boa dispersão das partículas de cimento melhorando o adensamento.

O RCMG foi substituído pelo cimento nas quantidades de $0 \%$ (S0), $5 \%$ (S5), 10\% (S10), 15\% (S15) e 20\% (S20) reduzindo o cimento na mesma proporção em peso mantendo o traço em massa de 1:0.8:1.7 (cimento + RCMG, areia, brita $0)$.

Para realizar o ensaio de compressão axial foram moldados 20 corpos de prova de cada grupo, totalizando 100 corpos de prova. Os corpos de prova foram moldados conforme NBR 5738 "Concreto: Procedimento para moldagem e cura de corpos de prova" (ABNT, 2016). A Tabela 4 ilustra a dosagem teórica por $\mathrm{m}^{3}$ do concreto produzido. 
Tabela 4: Dosagem do concreto

\begin{tabular}{ccccccc}
\hline Amostra & $\begin{array}{c}\text { Cimento } \\
\left(\mathrm{kg} / \mathrm{m}^{3}\right)\end{array}$ & $\begin{array}{c}\text { RCMG } \\
\left(\mathrm{kg} / \mathrm{m}^{3}\right)\end{array}$ & $\begin{array}{c}\text { Areia } \\
\left(\mathrm{kg} / \mathrm{m}^{3}\right)\end{array}$ & $\begin{array}{c}\text { Brita } \\
\left(\mathrm{kg} / \mathrm{m}^{3}\right)\end{array}$ & $\begin{array}{c}\text { Água } \\
\left(\mathrm{kg} / \mathrm{m}^{3}\right)\end{array}$ & $\begin{array}{c}\text { Aditivo } \\
\left(\mathrm{kg} / \mathrm{m}^{3}\right)\end{array}$ \\
\hline S0 (padrão) & 729 & - & 559 & 891 & 219 & 2.2 \\
\hline S5 & 692 & 37 & 559 & 891 & 219 & 2.2 \\
\hline S10 & 656 & 73 & 559 & 891 & 219 & 2.2 \\
\hline S15 & 619 & 110 & 559 & 891 & 219 & 2.2 \\
\hline S20 & 583 & 146 & 559 & 891 & 219 & 2.2 \\
\hline \hline
\end{tabular}

Fonte: Elaborado pelos autores (2021).

\subsection{Ensaio de absorção de água}

Nesta pesquisa utilizou-se como referência o Cimento Portland CPV ARIRS para confecção do corpo de prova que foram desmoldados 24 horas após a moldagem e para os corpos de prova com 1 dia de idade não foi realizado o ensaio de absorção de água, considerando esta idade como referência para as demais análises realizadas.

Após a desmoldagem, os corpos de prova foram imersos em água com temperatura média de $25^{\circ} \mathrm{C}$ e foram retiradas para ensaio de absorção com idades de 3, 7, 21 e 28 dias.

Os corpos de prova foram secos utilizando tela metálica para drenar a água durante aproximadamente 5 minutos e posteriormente foram secados com pano úmido e então foram pesados individualmente na condição saturada.

As peças permaneceram em repouso durante 4 horas e pesadas novamente para conferência do peso, sendo que nenhum dos corpos de prova ensaiados apresentou diferença de massa superior ou inferior a $0,5 \%$ em relação ao valor medido anteriormente.

Os corpos de prova foram então secados em estufa durante 24 horas com temperatura média de $105 \pm 1^{\circ} \mathrm{C}$ e, em seguida, foram pesadas novamente e não apresentaram diferença de massa superior a $0,5 \%$ em relação ao valor anterior, anotouse o valor da massa seca e o ensaio foi finalizado.

\subsection{Ensaio de compressão axial}

Foram moldados corpos de prova conforme a NBR 5738 (2016) no formato cilíndrico de $100 \mathrm{~mm}$ x $200 \mathrm{~mm}$, preenchidos com concreto em duas camadas sucessivas, cada uma delas recebendo 12 golpes com a haste de adensamento.

Os corpos de prova foram retificados e rompidos com as idades de 1 , 3, 7, 21 e 28 dias com aplicação de força contínua e sem choques até o ponto de ruptura.

O ensaio de compressão axial foi realizado conforme norma NBR 5739 "Concreto - Ensaio de compressão de corpos de prova cilíndricos" (ABNT, 2018).

\section{RESULTADOS E DISCUSSÃO}

\subsection{Caracterização da matéria prima}

A composição química do cimento e do RCMG foi avaliada através da técnica de espectroscopia por fluorescência de raios $X(F R X)$ e os resultados estão ilustrados na Tabela 5. 
Tabela 5: Composição química do cimento e RCMG

\begin{tabular}{lcccccccccccc}
\hline & $\mathrm{SiO}_{2}$ & $\mathrm{Al}_{2} \mathrm{O}_{3}$ & $\mathrm{MgO}$ & $\mathrm{K}_{2} \mathrm{O}$ & $\mathbf{C a O}$ & $\mathrm{Fe}_{2} \mathrm{O}_{3}$ & $\mathrm{Na}_{2} \mathrm{O}$ & $\mathrm{TiO}_{2}$ & $\mathrm{P}_{2} \mathrm{O}_{5}$ & $\mathrm{SO}_{3}$ & $\mathrm{MnO}$ & $\boldsymbol{\%} \mathrm{PF}$ \\
\hline CIMENTO & 22.65 & 4.10 & $\mathbf{3 . 5 0}$ & 0.35 & 60.9 & 3.35 & 0.55 & - & - & $\mathbf{1 . 7 0}$ & - & $\mathbf{1 . 6 0}$ \\
\hline $\mathbf{R C M G}$ & 69.10 & 18.73 & 2.71 & 2.31 & 2.05 & 1.91 & 1.61 & 0.34 & 0.33 & $\mathbf{0 . 1 2}$ & 0.04 & $\mathbf{1 6 . 8}$ \\
\hline $\begin{array}{l}\text { Limite NBR } \\
\mathbf{1 6 6 9 7}\end{array}$ & - & - & $\leq 6,5$ & - & - & - & - & - & - & $\leq 4,5$ & - & $\leq 6,5$ \\
\hline
\end{tabular}

Analisando a Tabela 5 os resultados corroboram com os principais componentes do cimento Portland, como o óxido de cálcio $(\mathrm{CaO})$, dióxido de silício $\left(\mathrm{SiO}_{2}\right)$, oxido de alumínio $\left(\mathrm{Al}_{2} \mathrm{O}_{3}\right)$, óxido de magnésio $(\mathrm{MgO})$, o óxido de ferro II $\left(\mathrm{Fe}_{2} \mathrm{O}_{3}\right)$, e Trióxido de enxofre $\left(\mathrm{SO}_{3}\right)$ em pequena quantidade, além de outros elementos em quantidades menores, como impurezas, os álcalis do cimento $\left(\mathrm{Na}_{2} \mathrm{O}\right.$, óxido de sódio e $\mathrm{K}_{2} \mathrm{O}$, óxido de potássio), e outras substâncias de menor importância como o óxido de titânio $\left(\mathrm{TiO}_{2}\right)$.

A norma regulamentadora NBR 16697 (ABNT, 2018) estabelece requisitos do teor de trióxido de enxofre (SO3) deve ser inferior a $4,0 \%$, pois é um composto instável, corrosivo e reage com a água, enquanto, o teor de óxido de magnésio
$(\mathrm{MgO})$ deve ser inferior a $6,5 \%$, pois em grandes quantidades no cimento, durante a hidratação, transforma-se lentamente em hidróxido de magnésio $\left(\mathrm{Mg}(\mathrm{OH})_{2}\right)$, expandindo e gerando tensões internas provocando trincas e fissuras.

Analisando a Tabela 5 o composto predominante no RCMG é dióxido de silício $\left(\mathrm{SiO}_{2}\right)$, seguido do óxido de alumínio $\left(\mathrm{Al}_{2} \mathrm{O}_{3}\right)$ e o óxido de cálcio ou cal livre $(\mathrm{CaO})$ que representam mais de $90 \%$ da composição química do composto.

A norma regulamentadora NBR 16697 (ABNT, 2018) estabelece que perda ao fogo (PF) após o aquecimento a $1000^{\circ} \mathrm{C}$ deve ser inferior a $6,5 \%$ de massa. Os resultados obtidos da caracterização física do cimento, areia, brita e RCMG estão ilustrados na Tabela 6.

Tabela 6: Composição química do cimento e RCMG

\begin{tabular}{lccccc}
\hline \multicolumn{1}{c}{ Características } & Cimento & Areia & Brita & RCMG & Unidade \\
\hline Modulo de finura & - & 1.800 & - & - & $\mathrm{kg} / \mathrm{dm}^{3}$ \\
\hline Material pulverulento & - & 1.300 & - & - & $\%$ \\
\hline Dimensão máx. característica & - & 2.360 & 6,3 & - & $\mathrm{mm}$ \\
\hline Massa unitária & - & 1.592 & 1.421 & - & $\mathrm{kg} / \mathrm{dm}^{3}$ \\
\hline Massa especifica & 3.252 & 2.621 & 2.822 & 2.920 & $\mathrm{~kg} / \mathrm{dm}^{3}$ \\
\hline Diâmetro médio da partícula & 0.050 & 0.600 & - & 0.012 & $\mathrm{~mm}$ \\
\hline Finura (resíduo peneira $\mathbf{7 5} \boldsymbol{\mu m})$ & 1.2 & - & - & - & $\%$ \\
\hline Tempo início de pega & 204 & - & - & - & $\mathrm{min}$ \\
\hline Tempo fim de pega & 275 & - & - & - & $\mathrm{min}$ \\
\hline Anidrido carbônico $\left(\mathbf{C O}_{2}\right)$ & 2.1 & - & - & - & $\%$ \\
\hline Permeabilidade Blaine & 4186 & - & - & - & $\mathrm{cm}^{2} / \mathrm{g}$ \\
\hline Resíduo insolúvel & 0.56 & - & - & - & $\%$ \\
\hline
\end{tabular}


Baseando-se nos resultados dos ensaios da Tabela 6, referente a caracterização física dos materiais desta pesquisa, é possível verificar que todos os resultados estão dentro dos limites estabelecidos nas normas regulamentadoras, entretanto, referente ao aglomerante proveniente de RCMG não existe valores estabelecidos em norma.

\subsection{Ensaio de compressão axial}

A Figura 1 ilustra os resultados médios obtidos no ensaio de compressão nas idades de 1,7 e 21 dias. Analisando a Figura 1, observou-se que o corpo de prova com adição de $20 \%$ de RCMG em substituição ao cimento foi o que apresentou o pior resultado entre os corpos de provas analisados, entretanto, os valores de resistência atendem os requisitos normativos exigidos pela NBR 9781 (ABNT, 2013), sendo indicados para utilização em locais de tráfego de pedestres, veículos leves e veículos comerciais de linha, pois apresentou resistência característica à compressão superior a $35 \mathrm{MPa}$ aos 28 dias.

A perda de resistência com a aumento da adição de RCMG pode ser explicada devido a absorção de maior quantidade de água que o aglomerante cimentício, aumentando a porosidade que influencia diretamente na perda de resistência à compressão axial, hipótese comprovada quando foi analisado o teor de absorção de água.

Conforme a Figura 2 percebeu-se que ocorreu um aumento de aproximadamente $60 \%$ na absorção de água aos 28 dias do corpo de prova $\mathrm{S} 20$ (20\% de RCMG) quando comparado ao corpo de prova padrão S0 que não teve adição de resíduo de corte de mármore e granito.

Figura 1: Resultados do ensaio da resistência à compressão axial

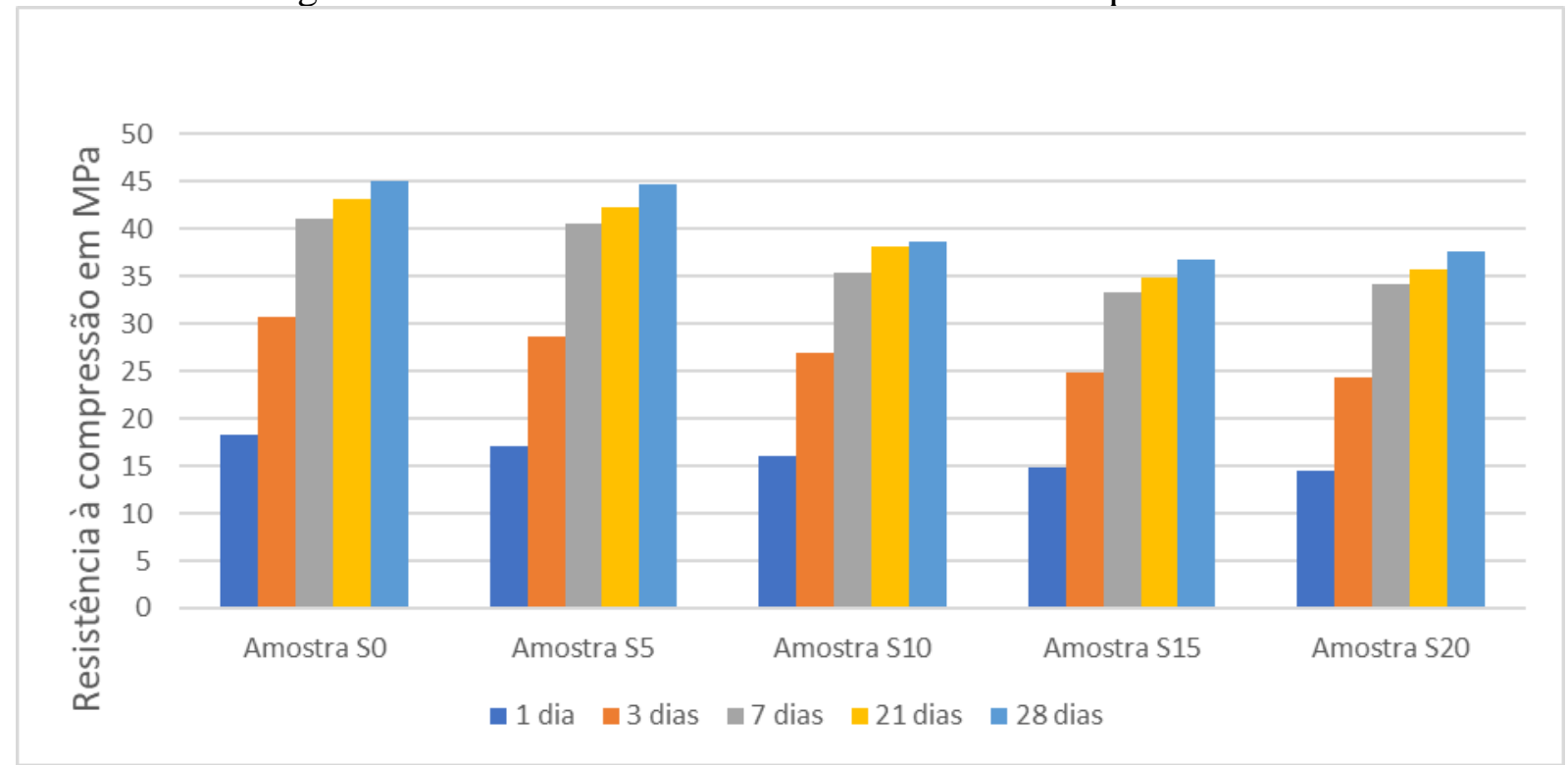

Fonte: Elaborado pelos autores (2021). 
Figura 2: Resultados do ensaio de absorção d'agua

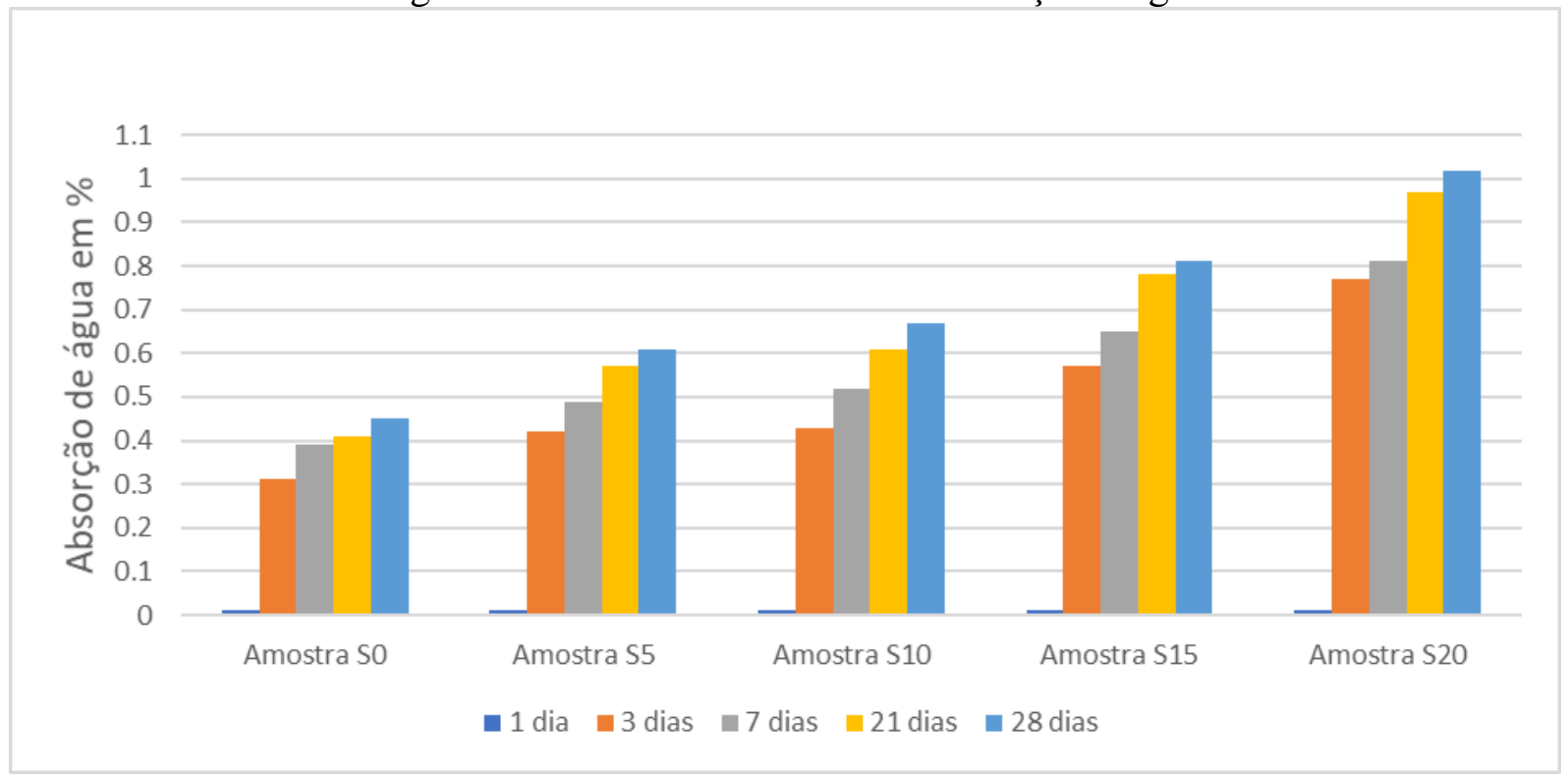

Fonte: Elaborado pelos autores (2021).

Os resultados desta pesquisa corroboram os estudos realizado por outros autores, como por exemplo Bilgin et al. (2012) que utilizaram RCMG na produção de tijolos industriais com proporções em massa variando de $0-80 \%$. Bilgin et al. (2012) observaram que teores de substituição de até $10 \%$ de RCMG não alteraram as propriedades mecânicas dos tijolos produzidos, comparado ao tijolo de referência, entretanto com adições superiores a $10 \%$ de RCMG ocorreu o aumento da absorção de água.

Krstulović et al. (1994) observaram que o RCMG melhorou a resistência à compressão axial de concreto e argamassas, principalmente em pequenas quantidades de resíduo, variando de 5 e $6 \%$ em relação à massa de cimento concluindo que este efeito é mais evidente se o RCMG for muito fino, como é o caso do resíduo utilizado na pesquisa.

Binici et al. (2007) utilizaram o RCMG como agregado graúdo e escória granulada de alto-forno como agregados miúdo na produção de concretos e perceberam a redução da penetração de cloretos em aproximadamente $70 \%$ obtendo melhor coesão entre os componentes da mistura (aditivos, cimento e agregados) indicando que os agregados de RCMG também podem ser utilizados para melhorar as propriedades mecânicas e a resistência química em relação ao concreto convencional.

A adição de resíduo de corte de granito (RCG) em concretos nas proporções de $10 \%$ a $20 \%$ em relação à massa de cimento, mantendo constante a relação água/cimento apresentaram maior coesão e consistência, melhorando o desempenho mecânico e aumentando a resistência à compressão axial entre $8 \%$ e $19,6 \%$ para os teores de $10 \%$ e $20 \%$, respectivamente.

No estudo realizado por Ergün (2011) $5 \%$ de cimento em peso foi substituído pelo resíduo de mármore e granito confirmando que substituição do cimento pelo resíduo melhorou as propriedades mecânicas dos concretos convencionais.

Conforme a literatura, outras pesquisas apresentaram resultados semelhantes quando o RCMG é substituo em teores de até $20 \%$, embora existam diversas variáveis divergentes que devem ser consideradas, a citar o traço do concreto, tipo de cimento utilizado e 
utilização do RCMG como agregado miúdo ou graúdo (Bilgin et al., 2012).

Todavia, os resultados desta pesquisa são corroborados por diversos autores, concluindo que a substituição do aglomerante pelo RCMG em até 20\% confirma a viabilidade técnica quanto ao uso em tráfego de pedestres, trânsito de veículos leves e comerciais, podendo diminuir os custos de produção e o impacto ambiental gerado pelo descarte de RCMG em aterros sanitários (Binici et al., 2007; Bilgin et al., 2012).

\section{CONCLUSÕES}

Dentre as várias formas de redução do impacto ambiental causado pela geração de resíduos, a que apresenta maiores vantagens é a reciclagem. A construção civil se apresenta como o setor com melhor potencial para a utilização dos resíduos devido aos inúmeros processos envolvendo diversos tipos de materiais. Além de contribuir para um maior desenvolvimento sustentável.

Quanto à resistência à compressão axial, a adição de RCMG não proporcionou ao concreto desempenho melhor que o do concreto de referência, entretanto o resultado final foi superior a $35 \mathrm{MPa}$ em todos os corpos de prova analisados.

Analisando os resultados de absorção de água notou-se que todos os corpos de prova apresentaram resultados satisfatórios, conforme NBR 9781 (ABNT, 2013) que estabelece a absorção máxima de $6 \%$ de água, sendo que os resultados oscilaram entre $0,45 \%$ e $1 \%$ de absorção de água, visto que o corpo de prova sem adição de resíduo apresentou o melhor resultado.

Conforme literatura os resíduos de corte de mármore e granito devem ser utilizados preferencialmente em substituição aos agregados graúdos e miúdos, entretanto, dependendo da utilização final do traço de concreto utilizado a utilização destes resíduos em substituição do aglomerante cimentício em até $20 \%$ pode apresentar resultados satisfatórios.

Em suma, a partir dos resultados obtidos no programa experimental, o uso do resíduo de corte de mármore e granito como adição em concretos e produção de pisos para pavimentação é viável tecnicamente para produção de pisos intertravados para pavimentação e tráfego de pedestres, veículos leves e veículos comerciais de linha.

\section{REFERÊNCIAS}

ARUNTAŞ, Hüseyin Y1lmaz; GÜRÜ, Metin; DAYI. Mustafa; TEKIN, Ilker. Utilization of waste marble dust as an additive in cement production. Materials and Design, v.31, 2010, 4039-4042 p. https://doi.org/10.1016/j.matdes.2010.03.036

AWAD, A.H.; EL-WAHAB, Abd.; ELGAMSY, Ramadan; ABDEL-LATIF, Hazen. A study of some thermal and mechanical properties of HDPE blend with marble and granite dust. Ain Shams Engineering Journal, Volume 10, Issue 2, 2019a, Pages 353-358, https://doi.org/10.1016/j.asej.2018.08.005

AWAD, A.H.; EL-WAHAB, Abd.; ELGAMSY, Ramadan; ABDEL-LATIF, Hazen. Assessment of mechanical properties of HDPE composite with addition of marble and granite dust, Ain Shams Engineering Journal, Volume 11, Issue 4, 2020, Pages 1211-1217, https://doi.org/10.1016/j.asej.2020.02.001

AWAD, A.H.; EL-WAHAB, Abd.; ELGAMSY, Ramadan; ABDEL-LATIF, Hazen. Mechanical behavior of PP reinforced with marble dust.

Construction and Building Materials, Volume 228, 2019b. https://doi.org/10.1016/j.conbuildmat.2019.1 16766 
AYDIN, Ertug; AREL, Hasan Sahan. High-volume marble substitution in cement-paste: Towards a better sustainability. Journal of Cleaner Production, v.237, 2019 http://doi.org/10.1016/j.jclepro.2019.117801

AZEVEDO, Afonso Rangel Garces; MARVILA, Markssuel Teixeira; BARROSO, Laimara da Silva; ZANELATO, Euzébio Barnabé; ALEXANDRE, Jonas; XAVIER, Gustavo de Castro; MONTEIRO, Sergio Neves. Effect of Granite Residue Incorporation on the Behavior of Mortars. Materials (Basel), 2019 http://dx.doi.org/10.3390/ma12091449

BAGHEL, Rupali; PANDEL, Upender; VASHISTHA, Apoorva. Manufacturing of sustainable bricks: Utilization of mill scale and marble slurry. Materials Today: Proceedings, v.26, 2020, 2136-2139 p. https://doi.org/10.1016/j.matpr.2020.02.460

BILGIN, N., YEPREM, H.A.; ARSLAN, S.; BILGIN, A.; GÜNAY, E.; MARŞOGLU, M. Use of waste marble powder in brick industry. Construction and Building Materials, v.29, 2012, 449$457 \mathrm{p}$.

http://doi.org/10.1016/j.conbuildmat.2011.10.011

BINICI, Hanifi; KAPLAN, Hasan; YILMAZ, Salih. Influence of marble and limestone dusts as additives on some mechanical properties of concrete.

Scientific Research and Essays, v.2, 2007, 372-379 p.

CHEN, J.J.; LI, B.H.; NG, P.L.; KWAN, A.K.H. Adding granite polishing waste as sand replacement to improve packing density, rheology, strength and impermeability of mortar. Powder Technology. v.364, 2020, 404-415 p. https://doi.org/10.1016/j.powtec.2020.02.012

CHIODI, Cid. O setor brasileiro de rochas ornamentais. Associação Brasileira da Indústria de Rochas Ornamentais, ABIROCHAS, 2018
ERGÜN, Ali. Effects of the usage of diatomite and waste marble powder as partial replacement of cement on the mechanical properties of concrete. Construction and Building Materials, v.25, 2011, 806-812 p.

http://doi.org/10.1016/j.conbuild mat.2010.07.002

GHALEHNOVI, Mansour; ROSHAN, Naein; HAKAK, Erfan; SHAMSABADI, Elyas Asadi; BRITO, Jorge. Effect of red mud (bauxite residue) as cement replacement on the properties of selfcompacting concrete incorporating various fillers. Journal of Cleaner Production, v.240, 2019

http://doi.org/10.1016/j.jclepro.2019.118213

GONÇALVES, Jardel Pereira. Utilização do resíduo de corte de granito (RCG) como adição para a produção de concretos. Porto Alegre, 2000. 135f. Dissertação, Universidade Federal do Rio Grande do Sul - UFRGS, 2000.

INCE, Ceren; HAMZA, Ali; DEROGAR, Shahram; BALL, Richard James.

Utilization of waste marble dust for improved durability and cost efficiency of pozzolanic concrete. Journal of Cleaner Production, v.270, 2020 http://doi.org/10.1016/j.jclepro.2020.122213

JAIN, Abhishek; GUPTA, Rajesh; CHAUDHARY, Sandeep. Sustainable development of self-compacting concrete by using granite waste and fly ash.

Construction and Building Materials, v.262, 2020

https://doi.org/10.1016/j.conbuildmat.2020.1 20516

KRSTULOVIĆ, P; KAMENK, N.; POPOVID, K. A new approach in evaluation of filler effect in cement I. Effect on strength and workability of mortar and concrete. Cement and Concrete Research, v. 24, 1994, 721-727 https://doi.org/10.1016/0008-8846(94)90197-X 
LI, L.G.; HUANG, Z.H.; TAN, Y.P.; KWAN, A.K.H.; CHEN, H.Y. Recycling of marble dust as paste replacement for improving strength, microstructure and ecofriendliness of mortar. Journal of Cleaner Production, V.210, 2019, 55-65

https://doi.org/10.1016/j.jclepro.2018.10.332

LI, L.G.; HUANG, Z.H.; TAN, Y.P.; KWAN, A.K.H.; LIU, F. Use of marble dust as paste replacement for recycling waste and improving durability and dimensional stability of mortar. Construction and Building Materials, v.166, 2018a, 423-432 http://doi.org/10.1016/j.conbuildmat.2018.01.154

LI, L.G.; WANG, Y.M.; TAN, Y.P.; KWAN, A.K.H.; LI, L.J. Adding granite dust as paste replacement to improve durability and dimensional stability of mortar. Powder Technology, v.333, 2018b, 269-276 p.

https://doi.org/10.1016/j.powtec.2018.04.055

LÓPEZ, Gemma Rojo; NUNES, Sandra; FONTEBOA, Belén González; ABELLA, Fernando Martínez. Quaternary blends of Portland cement, metakaolin, biomass ash and granite powder for production of selfcompacting concrete. Journal of Cleaner Production, Volume 266, 2020.

http://doi.org/10.1016/j.jclepro.2020.121666

MAHESH V. Patil; YOGESH D. Patil. Effect of copper slag and granite dust as sand replacement on the properties of concrete. Materials Today: Proceedings, 2021, 1666-1677p.

https://doi.org/10.1016/j.matpr.2020.10.029

MARTINI, Tcharllis Joao da Cunha; RODRÍGUEZ, Rubén Jesus Sánchez; SILVA, Fernanda Souza. Physical and mechanical evaluation of artificial marble produced with dolomitic marble residue processed by diamond-plated bladed gangsaws. Journal of Materials Research and Technology, v.7, 2018, 308-313 p. https://doi.org/10.1016/j.jmrt.2018.02.001
MASHALY, Ahmed; SHALABY, Basel; RASHWAN, Mohammed. Performance of mortar and concrete incorporating granite sludge as cement replacement.

Construction and Building Materials, v.169, 2018, 800-818

http://doi.org/10.1016/j.conbuildmat.2018.03.046

MATOS, Paulo Ricardo; SAKATA, Rafael Dors; GLEIZE, Philippe Jean Paul; BRITO, Jorge; REPETTE, Wellington Longuini. Eco-friendly ultra-highperformance cement pastes produced with quarry wastes as alternative fillers.

Journal of Cleaner Production, v.269, 2020, 41-54 p.

http://doi.org/10.1016/j.jclepro.2020.122308

MEDINA, G.; SÁEZ DEL BOSQUE, I.F.; FRÍAS, M.; SÁNCHEZ DE ROJAS, M.I.; MEDINA, C. Durability of new recycled granite quarry dust-bearing cements.

Construction and Building Materials, v.187, 2018, 414-425 p.

http://doi.org/10.1016/j.conbuildmat.2018.07.134

MESSAOUDA, Belouadah; RAHMOUNI, Zine El Abidine; TEBBAL, Nadia. Influence of the addition of glass powder and marble powder on the physical and mechanical behavior of composite cement. Procedia Computer Science, v.158, 2019, 366-375 p.

https://doi.org/10.1016/j.procs.2019.09.064

MONTANI, Carlo. Marble and stones in the world XXVIII report - international situation production and interchange. Casa di edizioni in carrara, v.18, 2017, 1-132 p.

NASCIMENTO, Anny Salonny; SANTOS, Cochiran Pereira; MELO, Fernanda Martins Cavalcante; OLIVEIRA, Vanessa Gentil Almeida; OLIVEIRA, Rosane Maria Pessoa Betanio; MACEDO, Zélia Soares; HERBET, Hebert Alves. Production of plaster mortar with incorporation of granite cutting wastes. Journal of Cleaner Production, v.265, 2020 https://doi.org/10.1016/j.jclepro.2020.121808 
NBR 11579: Cimento Portland Determinação do índice de finura por meio da peneira $75 \mu \mathrm{m}\left(\mathrm{n}^{\circ} \mathbf{2 0 0}\right)$. Associação Brasileira de Normas Técnicas, Rio de Janeiro, 2013.

NBR 12655: Concreto de cimento Portland - Preparo, controle, recebimento e aceitação - Procedimento, Associação Brasileira de Normas Técnicas, Rio de Janeiro, 2015.

NBR 16372: Cimento Portland e outros materiais em pó - Determinação da finura pelo método de permeabilidade ao ar (método de Blaine). Associação Brasileira de Normas Técnicas, Rio de Janeiro, 2015.

NBR 16605: Cimento Portland e outros materiais em pó - Determinação da massa específica. Associação Brasileira de Normas Técnicas, Rio de Janeiro, 2017.

NBR 16607: Cimento Portland Determinação dos tempos de pega. Associação Brasileira de Normas Técnicas, Rio de Janeiro, 2018.

NBR 16697: Cimento Portland Requisitos. Associação Brasileira de Normas Técnicas, Rio de Janeiro, 2018.

NBR 16916: Agregado miúdo Determinação da massa específica e massa específica aparente, Associação Brasileira de Normas Técnicas, Rio de Janeiro, 2021.

NBR 5738: Concreto de cimento Portland - Preparo, controle, recebimento e aceitação - Procedimento, Associação Brasileira de Normas Técnicas, Rio de Janeiro, 2016.

NBR 5739: Concreto - Ensaio de compressão de corpos de prova cilíndricos, Associação Brasileira de Normas Técnicas, Rio de Janeiro, 2016.
NBR 7211: Agregados para concreto Especificação. Associação Brasileira de Normas Técnicas, Rio de Janeiro, 2019.

NBR 9781: Peças de concreto para pavimentação - Especificação e métodos de ensaio. Associação Brasileira de Normas Técnicas, Rio de Janeiro, 2013.

NBR NM 14: Cimento Portland - Análise química - Método de arbitragem para determinação de dióxido de silício: óxido férrico: óxido de alumínio: óxido de cálcio e óxido de magnésio. Associação Brasileira de Normas Técnicas, Rio de Janeiro, 2012.

NBR NM 15: Cimento Portland - Análise química - Determinação de resíduo insolúvel. Associação Brasileira de Normas Técnicas, Rio de Janeiro, 2012.

NBR NM 16: Cimento Portland - Análise química - Determinação do anidrido sulfúrico. Associação Brasileira de Normas Técnicas, Rio de Janeiro, 2012.

NBR NM 18: Cimento Portland - Análise química - Determinação de perda ao fogo. Associação Brasileira de Normas Técnicas, Rio de Janeiro, 2012.

NBR NM 19: Cimento Portand Determinação de enxofre na forma de sulfeto. Associação Brasileira de Normas Técnicas, Rio de Janeiro, 2012.

NBR NM 20: Cimento Portland e suas matérias primas - Análise química Determinação de dióxido de carbono por gasometria. Associação Brasileira de Normas Técnicas, Rio de Janeiro, 2012.

SALMAN, Siddique; JEONG, Gook Jang; TRILOK, Gupta. Developing marble slurry as supplementary cementitious material through calcination: Strength and microstructure study. Construction and Building Materials, Volume 293, 2021. https://doi.org/10.1016/j.conbuildmat.2021.1 23474 
THAKUR, Anil Kumar; PAPPU, Asokan; THAKUR, Vijay Kumar. Resource efficiency impact on marble waste recycling towards sustainable green construction materials. Green and Sustainable Chemistry, 2018 https://doi.org/10.1016/j.cogsc.2018.06.005

TORRES, P.; FERNANDES, H.R.; OLHERO, S.; FERREIRA, J.M.F. Incorporation of wastes from granite rock cutting and polishing industries to produce roof tiles. Journal of the European Ceramic Society, v.29, 2009, 23-30 p. https://doi.org/10.1016/j.jeurceramsoc.2008. 05.045
ZANELATO, Euzebio; ALEXANDRE, Jonas; AZEVEDO, Afonso; MARVILA, Markssuel; XAVIER, Gustavo; MONTEIRO, Sergio. Evaluation of the Incorporation of Marble and Granite Residue in Coating Mortars. Advances in Powder and Ceramic Materials Science. The Minerals, Metals \& Materials Series. Springer, 2020, 101-108 p. https://doi.org/10.1007/978-3-030-36552-3_10 